\title{
Perfil Clínico-Epidemiológico das Neoplasias de Estômago Atendidas no Hospital do Câncer do Instituto do Câncer do Ceará, no Período 2000-2004

\author{
Clinical-Epidemiological Profile of Gastric Neoplasms in the Cancer Hospital \\ of Ceara's Cancer Institute, from the Period 2000-2004
}

\author{
Miren Maite Uribe Arregi', Débora Praciano Correia Férrer², Elaine Crystine Vieira de Assis ${ }^{3}$, Francisco Diego Silva de Paiva ${ }^{3}$, \\ Larissa Beatriz Guazzelli Sobral ${ }^{3}$, Nara Frota Andrée, Taciane Câmara da Silva ${ }^{3}$
}

\begin{abstract}
Resumo
A estimativa de casos novos de câncer de estômago para o Brasil em 2008 é de 14.080 entre homens e 7.720 nas mulheres. No Ceará, estima-se que será o segundo mais frequente em homens, e o terceiro, em mulheres. Para analisar o perfil clínico-epidemiológico dos pacientes com neoplasia de estômago, realizou-se estudo descritivo, transversal, quantitativo, de caráter retrospectivo, utilizando informações do Registro Hospitalar de Câncer e revisão de prontuário. A pesquisa incluiu 607 pacientes com neoplasia de estômago, atendidos no Hospital do Câncer do Instituto do Câncer do Ceará, de 2000 a 2004. Aproximadamente dois terços $(63,3 \%)$ foram do sexo masculino, a média de idade de 62,1 anos, para os homens, e de 57,9 para as mulheres. A maioria (63,5\%) possuía baixo nível educacional. A sublocalização mais frequente foi o corpo (43,2\%). Quanto ao tipo histológico, as neoplasias epiteliais representaram 85,7\%, sendo 70,0\% adenocarcinoma. Na classificação de Lauren, o tipo intestinal foi mais frequente. Nas neoplasias com estadiamento informado, a grande maioria (77,7\%) estava em estágio avançado (III ou IV). Há evidências de diferenças estatisticamente significativas ao comparar sublocalização e idade (proximal - média de idade maior), tipo histológico por idade (intestinal - média de idade maior), sexo (intestinal mais frequente em homens e difuso em mulheres) e nível educacional (intestinal ligado a nível educacional mais baixo). Conclui-se que as neoplasias de estômago compõem parte relevante do total de neoplasias atendidas nesse serviço e seguem o perfil descrito na literatura com relação à sublocalização anatômica, tipo histológico, estadiamento, idade, sexo e nível educacional.
\end{abstract}

Palavras-chave: Neoplasias gástricas; Perfil de saúde; Perfil de impacto da doença

${ }^{1}$ Médica, Mestre em Saúde Pública, Coordenadora do Registro Hospitalar de Câncer do Hospital do Câncer do Instituto do Câncer do Ceará (HC-ICC) ${ }^{2}$ Aluna de Graduação em Medicina da Universidade Federal do Ceará

${ }^{3}$ Alunos de Graduação em Medicina da Universidade Estadual do Ceará

Endereço para correspondência: Miren Maite Uribe Arregi. ICC - Registro Hospitalar de Câncer. Rua Papi Júnior, 1.222 - Rodolfo Teófilo - Fortaleza (CE), Brasil - CEP: 60430-230. E-mail: mirenuribe@yahoo.com.br 


\section{INTRODUÇÃO}

Nos últimos 70 anos, a incidência e a mortalidade mundiais do câncer gástrico caíram drasticamente devido, em grande parte, a um trunfo não planejado ${ }^{1,2,3,4,5,6}$. As explicações para o declínio são ainda hipóteses relacionadas à introdução da refrigeração; às mudanças nas técnicas de preservação de alimentos (usando menos sal e alimentos menos condimentados); ao consumo de frutas e de vegetais frescos; e à diminuição da prevalência da infecção por $\mathrm{H}$. pylori ${ }^{7}$. Hoje, com a estimativa de 1.000 .000 casos novos por ano, no mundo, encontra-se em quarto lugar ${ }^{5}$. A baixa sobrevida é refletida pela razão de mortalidade: incidência elevada, 70-90\% na maioria dos países, exceto Japão, onde é de $40 \%{ }^{8}$. Quase dois terços dos casos acontecem em países em desenvolvimento ${ }^{2}$, com áreas de incidência elevada no Leste da Ásia, no Leste Europeu e em partes da América do Sul e Central'5.

$\mathrm{O}$ número de casos novos de câncer de estômago estimados para o Brasil, no ano de 2008, é de 14.080 entre homens e de 7.720 nas mulheres. Esses valores correspondem ao risco estimado de 15 casos novos a cada 100 mil homens e oito para cada 100 mil mulheres. De acordo com as estimativas, no Ceará, será o segundo mais frequente em homens, e o terceiro, em mulheres ${ }^{4}$.

As informações do Registro de Câncer de Base Populacional de Fortaleza mostram que, no sexo masculino, o câncer gástrico representou, na década de 1990, 11,8\% do total de neoplasias, excluindo as de pele não melanoma; e, nas mulheres, 4,2\%. Em ambos os sexos, a incidência aumenta a partir de 35-40 anos e segue subindo apesar de mostrar ritmos diferentes?. Homens, idosos e grupos socioeconômicos desprovidos são os mais frequentemente afetados, independente da localização geográfica ${ }^{3,5,6}$.

A classificação histológica de Lauren divide os adenocarcinomas de estômago em dois tipos: intestinal e difuso. A razão intestinal/difuso costuma ficar ao redor de $1: 2$ e cresce com a idade ${ }^{2,10,11}$. O tipo intestinal predomina em áreas de risco elevado, especialmente em homens e em grupos de idade avançada ${ }^{12}$. O tipo difuso apresenta razão de casos entre homens e mulheres próxima à unidade, sendo mais frequente em pessoas abaixo dos 50 anos $^{2,11}$. O tipo intestinal é mais frequente em localização proximal do que distal ${ }^{13}$.

As lesōes do tipo intestinal são mais frequentes, diferenciadas, dependentes de fatores ambientais e associadas com a presença de lesões pré-cancerosas, como: gastrite crônica, atrofia gástrica, metaplasia intestinal e displasia ${ }^{14}$. O tipo difuso é pouco diferenciado, tem prognóstico ruim, apresenta-se em forma de tumores maiores ${ }^{10}$, costuma possuir um grau de penetração maior na parede gástrica e não está associado a lesões pré-cancerosas ${ }^{11,14}$. A invasão do fígado é recorrente e extensa no tipo intestinal, já o difuso ocasiona metástases peritoneais, pulmonares e ovarianas (tumores de Krukenberg) ${ }^{10,11}$.

O prognóstico do câncer gástrico avançado é reservado, com taxas de sobrevivência em cinco anos de $5 \%$ a $15 \%$, e está relacionado, entre outros fatores, com a localização anatômica do carcinoma. Dentro do estômago, dá-se, geralmente, nas formas piloro e antro, representando $50 \%$ a $60 \%$ dos casos; cárdia, $25 \%$; e o restante no corpo e fundo ${ }^{15}$. Os tumores predominam, tradicionalmente, no terço inferior ou médio ${ }^{16}$; mas, nos últimos 30 anos, tem sido descrito aumento na incidência de tumores proximais ${ }^{2}$.

Essa mudança tem sido observada principalmente nos países de baixo risco para câncer gástrico e nos homens ${ }^{2,3}$. A tendência particular do câncer de cárdia é indicação de que tem etiologia diferenciada, mas o aumento concomitante no período sugere que a neoplasia nessa sublocalização anatômica compartilha os mesmos fatores de risco, especialmente obesidade, refluxo gástrico e esôfago de Barrett subsequente ${ }^{8}$. Pesquisas em regióes com risco elevado de câncer de estômago e baixa incidência de esôfago de Barrett, como a China, mostram a necessidade de aprofundamento no estudo das neoplasias de cárdia ${ }^{17}$.

O objetivo deste trabalho foi analisar o perfil clínicoepidemiológico dos pacientes com neoplasia de estômago atendidos no Hospital do Câncer do Instituto do Câncer do Ceará (HC-ICC), no período 2000-2004.

\section{MATERIAL E MÉTODO}

Trata-se de um estudo descritivo, transversal, quantitativo e de caráter retrospectivo realizado no HC-ICC.

A população estudada é composta por todos os pacientes com câncer de estômago atendidos no HCICC, no período de 2000-2004.

A identificação das neoplasias foi realizada na base de dados do Registro Hospitalar de Câncer, fornecendo as principais informaçōes sobre o paciente e a doença. Dados complementares foram obtidos no prontuário do paciente.

As variáveis analisadas foram: sexo, idade, grau de instrução e profissão (nível socioeconômico), sublocalização anatômica do tumor, tipo histológico do tumor e estadiamento.

No estudo, foram observadas distribuições de frequências e realizadas comparações de médias (teste) e de variáveis categóricas (teste $\mathrm{X}^{2}$ e Fisher). O nível de significância observado foi de $5 \%$.

O trabalho foi aprovado pelo Comitê de Ética em Pesquisa do ICC, conforme a Resolução CNS/196/96. 


\section{RESULTADOS}

No período estudado, foram atendidos no HC-ICC 607 pacientes cuja neoplasia apresentava localização primária em estômago. Ao longo do período, foi observado aumento sustentado no número, passando de 87 casos, em 2000, para 147 casos, em 2004.

Aproximadamente dois terços $(63,3 \%)$ acometeram o sexo masculino. Mais da metade dos casos $(51,4 \%)$ concentra-se na faixa etária entre 55 e 74 anos (Tabela 1). A média de idade é de 62,1 anos, para os homens, e de 57,9 anos, para as mulheres, com medianas de 63 e 55 anos, respectivamente. A diferença observada nas médias foi estatisticamente significativa $(\mathrm{p}<0,001)$.

A maioria dos pacientes tinha baixo nível educacional, com $63,5 \%$ dos pacientes sendo analfabetos ou primeiro grau incompleto (Tabela 1). As ocupações mais citadas foram: agricultor $(29,9 \%)$ e doméstica $(28,9 \%)$. A base mais importante do diagnóstico foi a histologia do tumor primário (95,9\%), sendo que, em $97,3 \%$ dos casos, o diagnóstico foi microscópico (Tabela 1).

\section{SubloCALIZAÇÃO ANATÔMICA}

Importante percentual, $42,8 \%$, não teve a sublocalização informada; e, em $23,1 \%$ dos casos, a neoplasia comprometia dois ou mais locais contíguos e o local de origem não pôde ser determinado. Considerando somente as neoplasias cujo local de origem pôde ser definido, a sublocalização mais frequente foi o corpo $(43,2 \%)$, seguida pelo antro $(25,6 \%)$ e cárdia $(22,2 \%)$ (Tabela 1$)$.

As médias de idade, segundo sublocalização, apresentaram diferenças estatisticamente significativas $(\mathrm{p}<0,01)$ com valores de 66,6 anos, para os tumores localizados no cárdia; 69,5 anos, no fundo; 60,6 anos, no antro; 58,1 anos, no corpo; e, 56,5 anos, no piloro. Isso acontece também quando as neoplasias são classificadas em tumores proximais (corpo, fundo e cárdia) e distais (antropilóricas), com média de idade de 63,8 anos (mediana $=66,0$ ), no grupo dos tumores proximais, e de 58,0 anos (mediana $=60,0$ ), no grupo dos distais (Tabela 2).

Considerando unicamente os tumores com sublocalização especificada (347 casos), as distribuiçōes por sexo e por nível educacional não mostram diferença estatisticamente significativa.

\section{TIPO HISTOLÓGICO}

As neoplasias epiteliais foram a maioria $(85,7 \%)$. Dentre as não epiteliais, os linfomas foram os mais frequentes, representando $1,4 \%$ do total de casos. O tipo histológico mais comum, entre os epiteliais, foi o adenocarcinoma (70,0\%). Quando utilizada a Classificação de Lauren, o tipo intestinal foi o mais frequente (19,9\%) (Tabela 1).

Quando comparadas segundo o sexo, evidenciaramse diferenças estatisticamente significativas, nas quais o tipo intestinal é mais frequente em homens $(\mathrm{p}<0,01)$ (Gráfico 1). Também foi observada significância

Tabela 1. Características das neoplasias malignas de estômago, HC-ICC, 2000-2004

\begin{tabular}{|c|c|c|}
\hline Variável & Frequência & $\%$ \\
\hline \multicolumn{3}{|l|}{ Faixa etária } \\
\hline $\begin{array}{l}\text { Até } 40 \text { anos } \\
40-49 \\
50-59 \\
60-69 \\
70-79 \\
80 \text { ou }+\end{array}$ & $\begin{array}{c}59 \\
75 \\
121 \\
176 \\
132 \\
44\end{array}$ & $\begin{array}{c}9,7 \\
12,3 \\
19,9 \\
29 \\
21,7 \\
7,2\end{array}$ \\
\hline Sexo & Frequência & $\%$ \\
\hline $\begin{array}{l}\text { Homens } \\
\text { Mulheres }\end{array}$ & $\begin{array}{l}384 \\
223\end{array}$ & $\begin{array}{l}63,3 \\
36,7\end{array}$ \\
\hline Nível educacional & Frequência & $\%$ \\
\hline $\begin{array}{l}\text { Analfabeto ou } 1^{\circ} \text { grau } \\
\text { incompleto } \\
1^{\circ} \text { grau completo } \\
2^{\circ} \text { grau } \\
\text { Nível superior } \\
\text { Não informado }\end{array}$ & $\begin{array}{c}385 \\
59 \\
60 \\
31 \\
72\end{array}$ & $\begin{array}{c}63,5 \\
9,7 \\
9,9 \\
5,1 \\
11,9\end{array}$ \\
\hline $\begin{array}{l}\text { Sublocalização } \\
\text { anatômica }\end{array}$ & Frequência & $\%$ \\
\hline $\begin{array}{l}\text { Cárdia } \\
\text { Fundo } \\
\text { Antro } \\
\text { Corpo } \\
\text { Piloro } \\
\text { Não informada }\end{array}$ & $\begin{array}{c}77 \\
13 \\
89 \\
150 \\
18 \\
260\end{array}$ & $\begin{array}{c}12,7 \\
2,1 \\
14,7 \\
24,7 \\
3,0 \\
42,8\end{array}$ \\
\hline Localização & Frequência & $\%$ \\
\hline $\begin{array}{l}\text { Proximal } \\
\text { Distal } \\
\text { Não informada }\end{array}$ & $\begin{array}{l}179 \\
168 \\
260\end{array}$ & $\begin{array}{l}29,5 \\
27,7 \\
42,8\end{array}$ \\
\hline Tipo histológico & Frequência & $\%$ \\
\hline $\begin{array}{l}\text { Adenocarcinoma, SOE } \\
\text { Carcinoma intestinal } \\
\text { Carcinoma difuso } \\
\text { Carcinoma, SOE } \\
\text { Linfomas } \\
\text { Outros }\end{array}$ & $\begin{array}{c}220 \\
121 \\
84 \\
95 \\
9 \\
78\end{array}$ & $\begin{array}{c}36,3 \\
19,9 \\
13,8 \\
15,7 \\
1,4 \\
12,8\end{array}$ \\
\hline Estadiamento & Frequência & $\%$ \\
\hline $\begin{array}{l}0 \\
\text { I } \\
\text { II } \\
\text { III } \\
\text { IV } \\
\text { Não informado }\end{array}$ & $\begin{array}{c}1 \\
25 \\
52 \\
75 \\
196 \\
258\end{array}$ & $\begin{array}{c}0,2 \\
4,1 \\
8,6 \\
12,4 \\
32,3 \\
42,3\end{array}$ \\
\hline Total & 607 & 100,0 \\
\hline
\end{tabular}

Abreviatura SOE $=$ Sem outras especificaçōes 
Tabela 2. Distribuição das variáveis: sublocalização, tipo histológico (segundo Lauren) e estadiamento clínico, segundo média de idade, sexo e nível educacional, HC-ICC, 2000-2004

\begin{tabular}{|c|c|c|c|c|c|c|c|c|c|}
\hline \multirow{3}{*}{ Variável } & \multirow{3}{*}{$\begin{array}{l}\begin{array}{l}\text { Idade } \\
\text { (anos) }\end{array} \\
\text { Média }\end{array}$} & \multicolumn{4}{|c|}{ Sexo } & \multicolumn{4}{|c|}{ Nível educacional } \\
\hline & & \multicolumn{2}{|c|}{ Masculino } & \multicolumn{2}{|c|}{ Feminino } & \multicolumn{2}{|c|}{ Baixo } & \multicolumn{2}{|c|}{ Elevado } \\
\hline & & Frequência & $\%$ & Frequência & $\%$ & Frequência & $\%$ & Frequência & $\%$ \\
\hline \multicolumn{10}{|c|}{ Sublocalização } \\
\hline Proximal & 63,8 & 123 & 53,5 & 56 & 47,9 & 146 & 53,7 & 22 & 44,9 \\
\hline \multirow[t]{2}{*}{ Distal } & 58,0 & 107 & 46,5 & 61 & 52,1 & 126 & 46,3 & 27 & 55,1 \\
\hline & $P<0,001$ & $P>0,05$ & & & & $P>0,05$ & & & \\
\hline \multicolumn{10}{|c|}{ Tipo histológico segundo Lauren } \\
\hline Intestinal & 63,8 & 79 & 66,9 & 42 & 48,3 & 96 & 63,2 & 13 & 39,4 \\
\hline \multirow[t]{2}{*}{ Difuso } & 54,0 & 39 & 33,1 & 45 & 51,7 & 56 & 36,8 & 20 & 60,6 \\
\hline & $P<0,001$ & $P<0,01$ & & & & $P<0,01$ & & & \\
\hline \multicolumn{10}{|c|}{ Estadiamento } \\
\hline 1 & 60,2 & 16 & 8,6 & 6 & 5,1 & 16 & 7,7 & 6 & 6,2 \\
\hline II & 55,9 & 22 & 11,8 & 21 & 17,9 & 31 & 15,0 & 12 & 12,4 \\
\hline III & 58,1 & 48 & 25,7 & 21 & 17,9 & 46 & 22,2 & 23 & 23,7 \\
\hline \multirow[t]{2}{*}{ IV } & 56,5 & 101 & 54,0 & 117 & 59,0 & 114 & 55,1 & 56 & 57,7 \\
\hline & $P>0,05$ & $P>0,05$ & & & & $P>0,05$ & & & \\
\hline
\end{tabular}

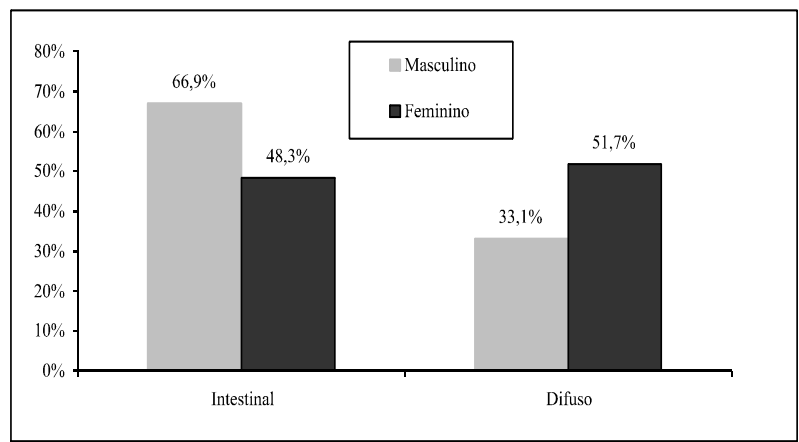

Gráfico 1. Distribuição das neoplasias de estômago, segundo tipo histológico (Lauren) e sexo, HC-ICC, 2000-2004

estatística ao comparar os tipos histológicos de Lauren segundo idade, em que os pacientes com o tipo intestinal tinham uma média de idade significativamente maior do que os pacientes com o tipo difuso $(\mathrm{p}<0,001)$ (Tabela 2$)$.

Foi observada relação inversa entre o nível educacional e o tipo de neoplasia, de acordo com a classificação de Lauren. Assim, o carcinoma tipo intestinal foi mais frequente entre pacientes de baixo nível educacional, enquanto o difuso era mais comum entre pacientes de nível educacional mais elevado (Gráfico 2).

\section{ESTADIAMENTO}

Um percentual importante dos casos $(42,3 \%)$ não teve o estágio informado. Entre os casos diagnosticados e tratados no Hospital, esse percentual cai para 29,1\%. Quando considerados os casos com estadiamento informado, mais da metade $(56,2 \%)$ apresentou estágio

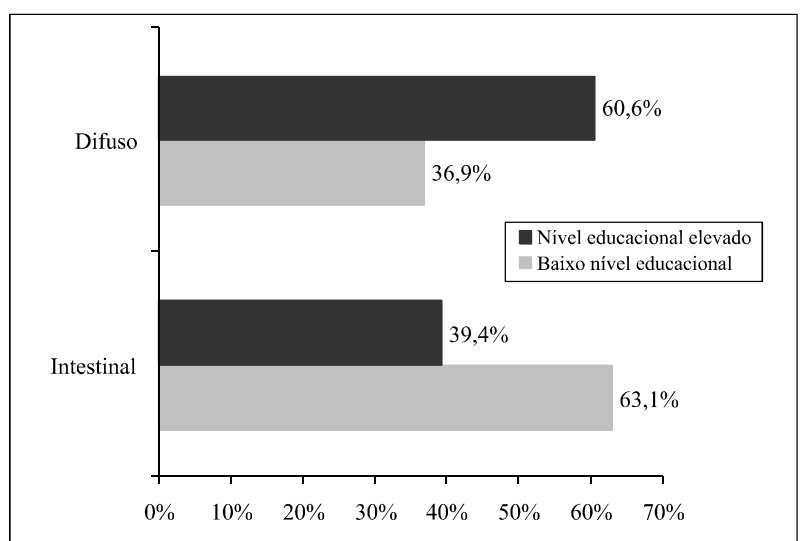

Gráfico 2. Distribuição dos carcinomas de estômago, pela Classificação de Lauren, segundo nível educacional, HC-ICC, 2000 2004

IV e a grande maioria estava em estágio avançado IIIIV $(77,7 \%)$ (Tabela 1$)$.

Tomando como base as neoplasias de estômago com estágio definido (exceto in situ), foram comparados o nível de estágio e a idade, não tendo sido observadas diferenças estatisticamente significativas $(p>0,05)$ nas médias de idade para cada grupo, assim como para o sexo e o nível educacional (Tabela 2).

\section{DISCUSSÃO}

No período estudado, foram atendidos no HC-ICC 607 pacientes com neoplasias de localização primária em estômago, representando a quinta neoplasia mais frequente no hospital. 


\section{SUBLOCALIZAÇÃO ANATÔMICA}

A distribuição das neoplasias gástricas apresenta variações de acordo com a sublocalização anatômica. As diferenças são observadas em populações com localização geográfica, características raciais e socioeconômicas distintas. Podem diferir em termos de suscetibilidade genética, perfil patológico, apresentação clínica e prognóstico, sugerindo tratar-se de doenças distintas com etiologias diferentes ${ }^{2}$.

Neste estudo, a sublocalização anatômica somente pôde ser definida para uma parte das neoplasias $(57,2 \%)$. Vários fatores contribuíram para a falta de definição, especialmente o fato das neoplasias serem diagnosticadas em estágio avançado. Dessa forma, no momento do diagnóstico, o tumor já avançou sobre várias áreas do estômago, fazendo impossível a identificação do local de origem da doença. Entre os que tiveram a sublocalização definida, a mais frequente foi o corpo $(43,2 \%)$, seguida pelo antro $(25,6 \%)$ e cárdia $(22,2 \%)$. Estudos nacionais e internacionais apresentam padrões semelhantes ${ }^{18}$.

Considerando a distribuição proximal/distal, no grupo que possuía classificação, a maioria dos casos $(51,6 \%)$ foi proximal (corpo, fundo e cárdia). Isto pode ser coerente com a situação geral descrita nos últimos 30 anos, que revela aumento na incidência de tumores proximais ${ }^{2,13}$, em contraposição com a maior frequência de sublocalização no terço inferior ou médio observada tradicionalmente ${ }^{16}$. Essa mudança tem sido verificada principalmente em países de baixo risco e entre homens ${ }^{2,3}$.

Estudos, especialmente baseados em registros de longa duração, como os do Norte da Europa, indicam que o aumento seria consequência, principalmente, de melhorias na classificação ${ }^{19}$. Alguns trabalhos já mostraram que os carcinomas de cárdia, fundo e corpo compartilham características que os diferenciam dos casos de antro, como o aspecto macroscópico, tamanho do tumor, profundidade da penetração parietal, invasão venosa, metástases nodais e o estágio $\mathrm{pTNM}^{20}$. Embora atribuam o aumento às melhorias na classificação ${ }^{19}$, os estudos mencionados apontam que nos EUA parece ser devido ao crescimento real ${ }^{21}$.

O fato de existir um percentual considerável de neoplasias em que a sublocalização original do tumor não pôde ser definida compromete essa avaliação, impossibilitando verificar se a mudança citada na literatura em relação ao aumento de tumores proximais é real em nossa população ou podendo afetar outros tipos de avaliações que relacionam sublocalização anatômica e sexo ou idade. Também deve ser considerado o fato de tratar-se de um estudo limitado a uma instituição hospitalar e que, portanto, inclui somente uma parte das neoplasias de estômago da população cearense.

\section{TIPO HISTOLÓGICO}

Os adenocarcinomas constituem cerca de $95 \%$ de todas as neoplasias de estômago. Dos outros 5\%, mais da metade é de linfomas ${ }^{12}$. Na presente pesquisa, as neoplasias epiteliais foram as mais frequentes $(85,7 \%)$. Dentre as não epiteliais, os linfomas foram os mais comuns, representando $1,4 \%$ do total de casos. Nesse estudo, o tipo histológico mais comum, entre os epiteliais, foi o adenocarcinoma (70,0\%). Corroborando com este estudo, Piso et al. ${ }^{13}$ mostrou que, entre os pacientes que tinham diagnóstico histologicamente confirmado de câncer gástrico, o adenocarcinoma foi o tipo histológico mais comum.

Entre os adenocarcinomas, a classificação de Lauren divide as neoplasias gástricas em dois tipos histológicos principais: intestinal e difuso. Esse sistema descreve os tumores com base na configuração microscópica e no padrão de crescimento $^{2}$, diferenciando duas entidades com etiologias e padrōes de progressão diferentes ${ }^{6}$, fundamentados em bases moleculares específicas que confirmam a relevância dessa classificação para o tratamento dessa patologia ${ }^{11}$.

O tipo intestinal mais frequente ${ }^{16}$ predomina em áreas de incidência elevada e é responsável pela variação internacional e queda de incidência das neoplasias de estômago ${ }^{2}$. O tipo difuso apresenta distribuição geográfica mais uniforme ${ }^{2}$. De forma coerente com o padrão de área de incidência elevada, o tipo intestinal foi o mais frequente neste trabalho, entre os que tiveram o padrão identificado segundo a classificação de Lauren.

A queda de incidência do câncer gástrico "endêmico", com patologia intestinal e localização no antro/corpo do estômago, e o aumento de incidência de tumores de cárdia aconteceram, especialmente, em países com baixas taxas de câncer gástrico, em associação a um nível socioeconômico mais elevado, em que o $\mathrm{H}$. pylori assume papel de menor importância ${ }^{3}$.

\section{IDADE}

Como em outras doenças, a incidência de câncer gástrico aumenta marcadamente com a idade ${ }^{12}$. Algumas pesquisas sugerem que o risco individual de câncer de estômago é estabelecido próximo ou abaixo dos 40 anos e que o aumento de sua expressão ocorre de forma previsível depois disso ${ }^{6}$. Segundo Crew e Neugut ${ }^{2}$, a incidência aumenta progressivamente com a idade, com pico de incidência entre 50 e 70 anos. Somente $2 \%$ a $9 \%$ de todos os pacientes com câncer gástrico têm menos de 40 anos de idade ${ }^{22}$. 
No presente estudo, mais da metade dos casos $(51,4 \%)$ concentrou-se na faixa etária entre 55 e 74 anos, mostrando uma tendência ao aumento da ocorrência dessa neoplasia à medida que a idade se eleva, e situandose na faixa de idade mais frequentemente relatada na literatura ${ }^{2,3,12}$, com picos em três grupos de idade: 50-59, 60-69 e 70-79 anos.

Assim como em outros trabalhos, as mulheres afetadas foram significativamente mais novas ( $m e ́ d i a=$ 57,9 anos) do que os homens (média $=62,1$ anos). A idade média de apresentação varia entre as sublocalizações. No câncer gástrico não cárdia, a incidência aumenta progressivamente com a idade, com pico de ocorrência entre 50 e 70 anos $^{2}$.

As médias de idade apresentaram diferenças significativas estatisticamente $(\mathrm{p}<0,01)$ com valores de 66,6 anos, para o grupo de indivíduos com tumores de cárdia; 69,5 anos, fundo; 60,6 anos, antro; 58,1 anos, corpo; e, 56,5 anos, piloro. Essas diferenças foram confirmadas quando comparados os grupos com tumores proximais $(63,8$ anos) e distais $(58,0$ anos) $(\mathrm{p}<0,001)$, de forma que, assim como em outros estudos, a média de idade é maior para o grupo de indivíduos com tumores proximais do que para aqueles com tumores médios ou distais ${ }^{13}$.

O tipo intestinal é mais frequente em homens idosos $^{10,11}$. O difuso é mais frequente em mulheres e pessoas abaixo dos 50 anos $^{11}$. A razão intestinal/difuso costuma ficar ao redor de 1:2 e cresce conforme aumenta a idade ${ }^{10}$.

\section{SEXO}

Diversos estudos mostram que os homens têm risco maior de desenvolver câncer de estômago do que as mulheres $^{6}$ e situam a relação homem/mulher entre 1,5$2,53,12$; se bem que, em populaçóes de alto risco, podem apresentar razões maiores, o que não é surpreendente se for considerada a predominância do tipo intestinal em áreas de risco elevado ${ }^{12}$. A relação entre os sujeitos deste estudo corresponde à citada, com aproximadamente dois terços $(63,3 \%)$ dos casos entre indivíduos do sexo masculino.

A literatura relata uma proporção masculino/ feminino para neoplasia gástrica não cárdia de aproximadamente 2:1. Em contraste, para carcinomas gástricos da cárdia, homens são cinco vezes mais afetados do que mulheres ${ }^{2}$. No grupo estudado, a distribuição por sexo não exibe diferenças estatisticamente significativas ( $p>0,05)$, mostrando, porém, a relação homem/mulher de 2,2 para tumores proximais e de 1,7 para os distais.

A relação homem/mulher é mais acentuada para o tipo intestinal. No tipo difuso, em áreas de baixo risco, a relação fica próxima de $1^{12}$. Os presentes dados mostraram uma diferença estatisticamente significativa quando comparados os tipos histológicos: intestinal e difuso, e sexo. Assim, o tipo intestinal foi mais frequente entre homens $(66,9 \%)$ e o difuso entre mulheres $(51,7 \%)$.

\section{NívEL SOCIOECONÔMICO}

$\mathrm{O}$ câncer gástrico é uma doença tipicamente de pobres ${ }^{12}$. Estudos baseados em dados de censos educacionais, de renda familiar ou de ocupação têm mostrado, de forma consistente, a associação entre neoplasia de estômago e o baixo nível socioeconômico ${ }^{2,3,12,19}$, em que a taxa em pessoas de baixo nível socioeconômico pode ser de duas a três vezes maior do que em classes mais afluentes ${ }^{23}$.

O nível socioeconômico per se não deve aumentar o risco de câncer de estômago, porém deve estar associado com vários fatores de risco, como o consumo de sal e a infecção por $\mathrm{H}$. pylori ${ }^{24}$. Neste trabalho, foram utilizados o nível de instrução e a ocupação como indicadores de renda. O nível de instrução foi baixo, com $63,5 \%$ dos pacientes analfabetos ou com primeiro grau incompleto. As ocupaçōes mais citadas foram a de agricultor $(29,9 \%)$ e doméstica $(28,9 \%)$.

Foi observada relação inversa entre o nível educacional e o tipo de neoplasia de acordo com a classificação de Lauren. Assim, o carcinoma tipo intestinal foi mais frequente entre pacientes de baixo nível educacional, enquanto o difuso era mais comum entre indivíduos de nível educacional mais elevado.

\section{ESTADIAMENTO}

O estadiamento é o fator prognóstico mais importante ${ }^{11}$. Os pacientes com estádios I e II têm melhor prognóstico, no entanto, mais de $50 \%$ dos doentes são diagnosticados nos estádios III e IV. Nesses estádios, a sobrevida após cinco anos é de 33,4\% e de $2 \%$, respectivamente ${ }^{25}$. Pelos dados deste trabalho, observa-se que ainda há um grande trabalho a realizar em relação ao diagnóstico precoce da doença.

A falta de informação nos prontuários hospitalares é limitação importante deste trabalho, no qual foram coletados dados sobre grande variedade de características dos tumores e pacientes. Partes como: história familiar, tabagismo, consumo de álcool, e outras foram excluídas pela proporção elevada de casos sem informação; e, nas variáveis descritas no trabalho, a falta de informação ou a inespecificidade impossibilitam conclusões mais firmes e podem ter comprometido as associaçôes estatísticas observadas. Este trabalho mostra dados sobre câncer gástrico que, na sua maioria, acompanham a literatura. O câncer gástrico continua sendo um problema 
importante no Ceará. O perfil descrito é similar ao observado nos países onde houve uma queda na incidência do câncer gástrico "endêmico", com patologia predominantemente intestinal e localização no antro e no corpo ( $1^{\mathrm{a}}$ e $2^{\mathrm{a}}$ localizaçóes anatômicas) e aumento da incidência de tumores na cárdia ( $3^{a}$ localização anatômica), embora nesses casos a prevalência do $\mathrm{H}$. pylori era menor por se tratar de países com um nível socioeconômico maior. Nesse sentido, é importante acompanhar as tendências de incidência e mortalidade para verificar mudanças em relação ao peso dessa neoplasia, que tem sido, tradicionalmente, uma das mais importantes no Estado do Ceará.

\section{AGRADECIMENTOS}

Agradecemos aos Drs. Cássio Cortéz e Marcelo Leite pelos esclarecimentos, assim como aos outros colegas do Serviço de Abdome e ao SAME do Hospital do Câncer do ICC.

\section{Potencial Conflito de Interesses: Nada a Declarar}

\section{REFERÊNCIAS}

1. Howson CP, Hiyama T, Wynder EL. The decline in gastric cancer: epidemiology of an unplanned triumph. Epidemiol Rev 1986; 8:1-27.

2. Crew KD, Neugut AI. Epidemiology of gastric cancer. World J Gastroenterol 2006 Jan 21;12(3):354-62.

3. Alberts SR, Cervantes A, Van de Velde CJ. Gastric cancer: epidemiology, pathology and treatment. Ann Oncol 2003;14 Suppl 2:ii, 31-6.

4. Brasil. Ministério da Saúde. Estimativa 2008: incidência do câncer no Brasil. Brasília: Ministério da Saúde; 2007.

5. American Cancer Society (ACS). Global Cancer Facts and Figures 2007. Estimated numbers of new cancer cases by world area. ACS. 2008. www.acs.org. Acessado em: 26 de maio de 2009.

6. Wachtel MS, Zhang Y, Chiriva-Internati M, Frezza EE. Different regression equations relate age to the incidence of Lauren types 1 and 2 stomach cancer in the SEER database: these equations are unaffected by sex or race. BMC Cancer 2006;15:6-65.

7. Latorre MR. Stomach cancer mortality in Brazil: from 1977 to 1989. Cad Saude Publica [serial online] 1997 [cited 2008 Jan 10];13 Suppl 1:[12 screens]. Available from: URL: http://www.scielosp.org

8. Plummer JM, Franceschi S, Munoz N. Epidemiology of gastric cancer. IARC Sci Publ 2004;157:311-26.

9. Ceará. Secretaria de Saúde do Estado do Ceará. Incidência de câncer em Fortaleza: dados do Registro de Câncer de
Base Populacional - RCBP, 1990-1999. 2007. [acesso em: 10 jan 2008] Disponível em: URL: http:// www.saude.ce.gov.br

10. Wu X, Chen VW, Ruiz B, Andrews P, Su LJ, Correa P. Incidence of esophageal and gastric carcinomas among American Asians/Pacific Islanders, whites, and blacks: subsite and histology differences. Cancer 2006;106(3): 683-92.

11. Zheng H, Takahashi H, Murai Y, Cui Z, Nomoto K, Miwa $S$, et al. Pathobiological characteristics of intestinal and diffuse-type gastric carcinoma in Japan: an immunostaining study on the tissue microarray. J Clin Pathol 2007 Mar;60(3):273-7.

12. Nomura A. Stomach cancer. In: Nomura D, Fraumeni JFJ, editors. Cancer epidemiology and prevention. New York: Oxford University Press; 1996. p. 707-24.

13. Piso P, Werner U, Lang H, Mirena P, Klempnauer J. Proximal versus distal gastric carcinoma-what are the differences? Ann Surg Oncol 2000 Aug;7(7):520-5.

14. César ACG, Silva AR, Tajara EH. Fatores genéticos e ambientais envolvidos na carcinogênese gástrica. Arq Gastroenterol 2002; 39(4): 253-9.

15. Liu Y, Kaneko S, Sobue T. Trends in reported incidences of gastric cancer by tumour location, from 1975 to 1989 in Japan. Int J Epidemiol 2004; 33:808-15.

16. Csendes A. Adenocarcinoma of the stomach: a review of 35 years and 1,710 cases. World J Surg 1991 JulAug; 15(4):558.

17. Wang LD, Zheng S, Zheng ZY, Casson AG. Primary adenocarcinomas of lower esophagus, esophagogastric junction and gastric cardia: in special reference to China. World J Gastroenterol 2003 Jun;9(6):1156-64.

18. De Stefani E, Boffetta P, Mendilaharsu M, Carzoglio J, Deneo-Pellegrini H. Dietary nitrosamines, heterocyclic amines, and risk of gastric cancer: a case-control study in Uruguay. Nutr Cancer 1998;30(2):158-62.

19. Ekström AM, Eriksson M, Hansson LE, Lindgren A, Signorello LB, Nyrén O, et al. Occupational exposures and risk of gastric cancer in a population-based casecontrol study. Cancer Res 1999 Dec 1;59(23):5932-7.

20. Pinto-De-Sousa J, David L, Seixas M, Pimenta A. Clinicopathologic profiles and prognosis of gastric carcinomas from the cardia, fundus/body and antrum. Dig Surg 2001;18(2):102-10.

21. Devesa S, Fraumeni JFJ. The rising incidence of gastric cardia cancer. J Natl Cancer Inst 1999;91:747-9.

22. Koea JB, Karpeh MS, Brennan MF. Gastric cancer in young patients: demographic, clinicopathological, and prognostic factors in 92 patients. Ann Surg Oncol 2000 Jun;7(5):346-51.

23. Muñoz N, Franceschi S. Epidemiology of gastric cancer and perspectives for prevention. [cited 2008 Feb 13]. Salud Publica Mex [serial online] 1997; 39(4):[13 screens]. Available from: URL: http://www.scielosp.org 
24. Nishimoto IN, Hamada GS, Kowalski LP, Rodrigues JG, Iriya K, Sasazuki S, et al. Risk Factors for Stomach Cancer in Brazil: a Case-control Study among NonJapanese Brazilians in São Paulo. Jpn J Clin Oncol 2002; 32:277-83.
25. Mandorwski S, Gomes LL, Manoukian FN. CA72-4 e CEA no soro e no lavado peritonial de doentes com câncer gástrico. [cited $2008 \mathrm{Feb}$ 13]; Arq Gastroenterol [serial online] $2002 \mathrm{Mar}$ 39(1):[5 screens]. Available from: URL: http://www.scielo.br

\section{Abstract}

In 2008, it is estimated that there will be 14,080 new cases of stomach cancer among men and 7,720 among women in Brazil. In the state of Ceará, it is supposed that this will be the second most common type of cancer among men, and the third among women. To review the clinical and epidemiological profile of patients with stomach cancer, a descriptive, cross-quantitative and retrospective research was done, using data from the Hospital-Based Cancer Registry and by reviewing medical records. The research includes 607 patients with stomach cancer that were treated at Ceará's Cancer Institute Cancer Hospital from 2000 to 2004. Approximately two thirds $(63.3 \%)$ are male, whose average age is 62 years for men, and 58 years for women. Most of them $(63.5 \%)$ have a low educational level. The most frequent subsite is the body (43.2\%). As for the histological type, the epithelial neoplasms represent $85.7 \%, 70.0 \%$ of which is adenocarcinoma. In Lauren's classification, the intestinal type of this cancer is more common. In neoplasms with informed staging, the majority $(77.7 \%)$ is in the advanced stage (III or IV). There is evidence of statistically significant differences when comparing subsites and age (proximal - average among the elder), histological type by age (intestinal - average among the elder), gender (intestinal- more frequent in men and diffused in women) and educational level (intestinal was linked to a lower educational level). Stomach neoplasms comprise a significant percentage of tumors seen in the hospital and the data follows the profile described in the literature regarding the subsite, histological type, cancer stage, age, gender and educational level.

Key words: Stomach neoplasms; Health profile; Sickness impact profile 